\title{
Identification and characterization of critical genes associated with tamoxifen resistance in breast cancer
}

\author{
Kai Zhang ${ }^{\text {Equal first author, } 1}{ }^{1}$, Kuikui Jiang ${ }^{\text {Equal first author, } 2}{ }^{2}$, Ruoxi Hong ${ }^{2}$, Fei Xu ${ }^{2}$, Wen Xia ${ }^{2}$, Ge Qin ${ }^{2}$, Kaping Lee ${ }^{2}$, Qiufan \\ Zheng $^{2}$, Qianyi Lu ${ }^{2}$, Qinglian Zhai ${ }^{2}$, Shusen Wang ${ }^{\text {Corresp. } 2}$ \\ ${ }^{1}$ Key laboratory of Carcinogenesis and Translational Research (Ministry of Education/Beijing), Laboratory of Molecular Oncology, Peking University Cancer \\ Hospital \& Institute, Beijing, China \\ 2 Sun Yat-sen University Cancer Center; State Key Laboratory of Oncology in South China; Collaborative Innovation Center for Cancer Medicine, \\ Guangzhou, China \\ Corresponding Author: Shusen Wang \\ Email address: wangshs@sysucc.org.cn
}

Background. Tamoxifen resistance in breast cancer is an unsolved problem in clinical practice. The aim of this study was to determine the potential mechanisms of tamoxifen resistance through bioinformatics analysis.

Methods. Gene expression profiles of tamoxifen-resistant MCF-7/TR and MCF-7 cells were acquired from the Gene Expression Omnibus (GEO) dataset GSE26459, and differentially expressed genes (DEGs) were detected with R software. We conducted Gene Ontology (GO) and Kyoto Encyclopedia of Genes and Genomes (KEGG) pathway enrichment analyses using Database for Annotation, Visualization and Integrated Discovery (DAVID). A protein-protein interaction (PPI) network was generated, and we analyzed hub genes in the network with the Search Tool for the Retrieval of Interacting Genes (STRING) database. Finally, we used siRNAs to silence the target genes and conducted the MTS assay.

Results. We identified 865 DEGs, 399 of which were upregulated. GO analysis indicated that most genes are related to telomere organization, extracellular exosomes, and binding-related items for protein heterodimerization. PPI network construction revealed that the top 10 hub genes-ACLY, HSPD1, PFAS, GART, TXN, HSPH1, HSPE1, IRAS, TRAP1, and ATIC-might be associated with tamoxifen resistance. Consistently, RT-qPCR analysis indicated that the expression of these 10 genes was increased in MCF-7/TR cells comparing with MCF-7cells. Four hub genes (TXN, HSPD1, HSPH1andATIC) were related to overall survival (OS) in patients who accepted tamoxifen. In addition, knockdown of HSPH1 by siRNA may lead to reduced growth of MCF-7/TR cell with a trend close to significance $(P=0.07)$, indicating that upregulation of $\mathrm{HSPH} 1$ may play a role in tamoxifen resistance.

Conclusion. This study revealed a number of critical hub genes that might serve as therapeutic targets in breast cancer resistant to tamoxifen and provided potential directions for uncovering the mechanisms of tamoxifen resistance. 


\section{Identification and characterization of critical genes}

G। 3 associated with tamoxifen resistance in breast cancer 4

5

Kai Zhang ${ }^{1 \#}$, Kuikui Jiang ${ }^{2 \#}$, Ruoxi Hong ${ }^{2}$, Fei Xu ${ }^{2}$, Wen $\mathrm{Xia}^{2}$, Ge Qin², Kaping Lee², Qiufan Zheng', Qianyi Lu², Qinglian Zhai ${ }^{2}$, Shusen Wang ${ }^{2 *}$

${ }^{1}$ Key laboratory of Carcinogenesis and Translational Research (Ministry of Education/Beijing), Laboratory of Molecular Oncology, Peking University Cancer Hospital \& Institute, Beijing 100142, China

2 Sun Yat-sen University Cancer Center; State Key Laboratory of Oncology in South China; Collaborative Innovation Center for Cancer Medicine, Guangzhou 510060, China.

\# The two authors contributed equally to this work as first authors.

Corresponding Authors:

Sun Yat-sen University Cancer Center; State Key Laboratory of Oncology in South China; Collaborative Innovation Center for Cancer Medicine, 651 Dongfeng Road East, Guangzhou, 21 510060, China.

Email address: wangshs@sysucc.org.cn

23 


\section{Abstract}

42 Background. Tamoxifen resistance in breast cancer is an unsolved problem in clinical practice.

43 The aim of this study was to determine the potential mechanisms of tamoxifen resistance through

44 bioinformatics analysis.

45 Methods. Gene expression profiles of tamoxifen-resistant MCF-7/TR and MCF-7 cells were 46 acquired from the Gene Expression Omnibus (GEO) dataset GSE26459, and differentially 47 expressed genes (DEGs) were detected with R software. We conducted Gene Ontology (GO) and 48 Kyoto Encyclopedia of Genes and Genomes (KEGG) pathway enrichment analyses using 49 Database for Annotation, Visualization and Integrated Discovery (DAVID). A protein-protein 50 interaction (PPI) network was generated, and we analyzed hub genes in the network with the 51 Search Tool for the Retrieval of Interacting Genes (STRING) database. Finally, we used siRNAs to silence the target genes and conducted the MTS assay. Results. We identified 865 DEGs, 399 of which were upregulated. GO analysis indicated that most genes are related to telomere organization, extracellular exosomes, and binding-related items for protein heterodimerization. PPI network construction revealed that the top $10 \mathrm{hub}$ genes-ACLY, HSPD1, PFAS, GART, TXN, HSPH1, HSPE1, IRAS, TRAP1, and ATIC-might be associated with tamoxifen resistance. Consistently, RT-qPCR analysis indicated that the expression of these 10 genes was increased in MCF-7/TR cells comparing with MCF-7cells. Four hub genes (TXN, HSPD1, HSPHI and $A T I C$ ) were related to overall survival (OS) in patients who accepted tamoxifen. In addition, knockdown of HSPH1 by siRNA may lead to reduced growth of $\mathrm{MCF}-7 / \mathrm{TR}$ cell with a trend close to significance $(\mathrm{P}=0.07)$, indicating that upregulation of HSPH1 may play a role in tamoxifen resistance. the mechanisms of tamoxifen resistance. 


\section{Introduction}

82 About $70 \%$ of breast cancer cases worldwide are estrogen receptor (ER) positive. ER-positive

83

84

85

86

87

88

89

90

91

92

93

94

95

96

97

98

99

100

101

102

103

104

105

106

107

108

109

110

111

112

113

114

115

116

117

118

119

120

breast cancer is an endocrine-dependent disease, and ER plays a vital role in the metastasis, occurrence and progression of this disease (Baum et al., 2002; Torre et al., 2015; Turner et al., 2017). Tamoxifen, a selective estrogen receptor modulator, binds to ER $\alpha$ and significantly suppresses the estrogen-induced growth of mammary epithelial cells(Early Breast Cancer Trialists' Collaborative Group, 1998). However, approximately one-third of ER-positive breast cancers inevitably develop tamoxifen resistance, which leads to an unfavorable prognosis and remains a crucial clinical challenge to effective treatment (Ring \& Dowsett, 2004). Thus, it is critical to elucidate the underlying factors that cause tamoxifen resistance, identify improved treatment strategies, and develop effective regimens for this disease (Shang \& Brown, 2002; Gutierrez et al., 2005).

In recent years, many curative measures attempting to overcome tamoxifen resistance have been explored (Ring \& Dowsett, 2004; Viedma-Rodriguez et al., 2014), and previous investigations have highlighted the use of specific molecular targeted drugs (Normanno et al., 2005). However, due to the unclear mechanisms, analyses of such approaches are still in the early stages and the treatment effects are not satisfactory (Nass et al., 2015; Zhao et al., 2017). Overall, the molecular mechanisms of tamoxifen resistance have not been determined. Exploration into the underlying factors of tamoxifen resistance would affect targeting strategies that might overcome tamoxifen resistance and enhance clinical results (Rondon-Lagos et al., 2016). Accordingly, identification of the genes related to tamoxifen resistance with powerful gene sequencing technologies, which may elucidate the mechanisms of tamoxifen resistance and help identify new curative strategies, is urgently needed (Katzenellenbogen \& Frasor, 2004). However, few studies have been done on the key genes associated with tamoxifen resistance in breast cancer through bioinformatics analysis.

Gene microarray technology is an enhanced high-throughput method to effectively analyze gene expression profiles. Thus, gene microarrays have been comprehensively used to explore the underlying regulatory networks involved in different types of cancer and have important clinical applications in improving clinical diagnoses and discovering new drug targets (Zhang et al., 2017). This technology can also identify thousands of differentially expressed genes (DEGs) related to different biological processes (BPs), cellular components (CCs), molecular functions (MFs), and different signaling pathways of cancer. Moreover, bioinformatics methods allow for comprehensive analysis of large amounts of data derived from microarrays. Given the false positives and heterogeneity of different microarray results, we processed one microarray dataset, GSE26459, to obtain DEGs between the ER-positive breast cancer cell line MCF-7 (cell line sensitive to tamoxifen) and MCF-7/TR (cell line with acquired tamoxifen resistance). These findings combined with the bioinformatics results identified important signaling pathways and hub genes involved in breast cancer tamoxifen resistance. Through survival analysis, we found that ATIC, HSPDI, HSPH1 and TXN were related to survival. Further knockdown of target genes by siRNAs indicated that upregulation of HSPH1 is likely to play a role in tamoxifen resistance.

Peer) reviewing PDF | (2020:07:50749:1:2:NEW 2 Nov 2020) 
121 Taken together, our results provide a valuable perspective into the mechanisms of tamoxifen 122 resistance, identify possible candidate biomarkers, and suggest potential therapeutic drugs for 123 overcoming tamoxifen resistance.

124

\section{Materials \& Methods}

126

127

128

129

130

131

132

133

134

135

136

137

138

139

140

141

142

143

144

145

146

147

148

149

150

151

152

153

154

155

156

157

158

159

\section{Cell culture}

MCF-7 and tamoxifen-resistant MCF-7 (MCF-7/TR) cell lines were obtained from the Breast Tumor Center, Sun Yat-sen Memorial Hospital, Sun Yat-sen University (Zhu et al., 2018). Tamoxifen-resistant MCF-7 (MCF-7/TR) cells were established as formerly reported (Badia et al., 2000; Knowlden et al., 2003). MCF-7 cells were cultured in DMEM (Gibco, USA) with 10\% FBS (Gibco, USA). Tamoxifen-resistant MCF-7 (MCF-7/TR) cells were cultured in DMEM (Gibco, USA) supplemented with 10\% FBS (Gibco, USA) and $1 \mu \mathrm{M}$ tamoxifen.

\section{Identification of DEGs}

To identify DEGs in tamoxifen-resistant MCF-7/TR cells and tamoxifen-sensitive MCF-7 cells, we obtained raw public gene expression data (GSE26459) from the GEO database. This dataset contains information for MCF-7 and MCF-7/TR cells. These data were analyzed through R software with the affy and limma Bioconductor packages (Ritchie et al., 2015). To identify tamoxifen resistance-related DEGs between MCF-7/TR and MCF-7 cells, Student's $t$ test was carried out and statistical significance was set at $\mathrm{P}<0.05$ with a fold change $\geq 1.5$.

\section{DEG Gene Ontology and pathway enrichment analysis}

To determine the biological functions and signaling pathways related to the DEGs, we submitted the data to Database for Annotation, Visualization and Integrated Discovery (DAVID), which is an online tool for gene annotation, function visualization and large volume data integration, including GO functional analysis and Kyoto Encyclopedia of Genes and Genomes (KEGG) pathway analysis (Schmid \& Blaxter, 2008; Huang, Sherman \& Lempicki, 2009). GO classifications include $\mathrm{CC}, \mathrm{BP}$ and $\mathrm{MF}$ groups and $\mathrm{P}<0.001$ was considered significant.

\section{Protein-protein interaction (PPI) network construction by STRING}

To evaluate PPI network information for the significant DEGs, we analyzed the 865 DEGs by the online database Search Tool for the Retrieval of Interacting Genes (STRING) to predict interactions among them (Szklarczyk et al., 2015). A combined score $>0.7$ was considered a high confidence score. These DEGs with many associations with other genes have critical relationships in the PPI interaction network. The top 15 upregulated DEGs in the PPI network were visualized through Cytoscape software (version 3.3.0) (Shannon et al., 2003). CytoHubba was employed to detect hub proteins according to their associations with other proteins by using Cytoscape software (version 3.3.0) (Chin et al., 2014).

\section{RT-qPCR assay}

The primer sequences used were as follows: ATP Citrate Lyase ( $A C L Y)$ forward, 5'TCGGCCAAGGCAATTTCAGAG-3' and reverse, 5'-CGAGCATACTTGAACCGATTCT-3'; Heat Shock Protein Family D (Hsp60) Member 1 (HSPD1) forward, 5- 
160

161

162

163

164

165

166

167

168

169

170

171

172

173

174

175

176

177

178

179

180

181

182

183

184

185

186

187

188

189

190

191

192

193

194

195

196

197

ATGCTTCGGTTACCCACAGTC-3' and reverse, 5'-AGCCCGAGTGAGATGAGGAG-3'; Phosphoribosylformylglycinamidine Synthase (PFAS) forward, 5'-

CGAGCATACTTGAACCGATTCT-3' and reverse, 5'-GTAGCACAGTTCAGTCTCGAC-3'; Phosphoribosylglycinamide Formyltransferase (GART) forward, 5'GGAATCCCAACCGCACAATG-3' and reverse, 5'-AGCAGGGAAGTCTGCACTCA-3'); Thioredoxin $(T X N)$ forward, 5'-GTGAAGCAGATCGAGAGCAAG-3' and reverse, 5'CGTGGCTGAGAAGTCAACTACTA-3'; Heat Shock Protein Family H (Hsp110) Member 1 (HSPH1) forward, 5'-ACAGCCATGTTGTTGACTAAGC-3' and reverse, 5'GCATCTAACACAGATCGCCTCT-3'; Heat Shock Protein Family E (Hsp10) Member 1 (HSPE1) forward, 5'-ATGGCAGGACAAGCGTTTAGA-3' and reverse, 5'-

TGGAAGCATAATGCCTCCTTTG-3'; TNF Receptor Associated Protein 1 (TRAPI) forward, 5'-AGGACGACTGTTCAGCACG-3' and reverse, 5'-CCGGGCAACAATGTCCAAAAG-3';

Nischarin (IRAS) forward, 5'-CAACCT ACGGATGACTCGTGCTT-3' and reverse, 5'-

TTTCTCCCTGACGGTCCCACTT-3'; 5-Aminoimidazole-4-Carboxamide Ribonucleotide (ATIC) forward, 5'-ACCTGACCGCTCTTGGTTTG-3' and reverse, 5'-

TACGAGCTAGGATTCCAGCAT-3'. Glyceraldehyde-3-Phosphate Dehydrogenase (GAPDH) forward, 5'-GGAGCGAGATCCCTCCAAAAT-3' and reverse, 5'GGCTGGGTGCATCATTCTCATGG-3'.

\section{Survival analysis of hub genes}

We analyzed the overall survival of breast cancer patients stratified by the expression of 10 hub genes (high and low) using the Kaplan-Meier Plotter (http://www.kmplot.com), which is an online tool accumulating data on the gene expression and survival of breast cancer patients.

\section{Cell viability assay}

The breast cancer cell lines were plated in 96-well plates (2,000-5000 cells/well) and treated with escalating doses of TAM as single agent. According to the manufacturer's instructions, MTS assays (Promega) were conducted to detect the proliferation ability of tumor cells. The half maximal inhibitory concentrations (IC50) were calculated by GraphPad Prism version 7. Data were presented as the mean $\pm \mathrm{SD}$ of three independent experiments.

\section{siRNA transfection}

MCF-7/TR cells were transfected with specific siRNA or negative control siRNA using RNAi MAX and the negative control were designed by ribo. These cells were cultured for 24-48 hours in a $\mathrm{CO}_{2}$-containing chamber at $37{ }^{\circ} \mathrm{C}$ before being used for cell-based assays.

\section{Statistical analysis}

All data were statistically analyzed using Student's $t$ test with GraphPad Prism 7 software. The internal GAPDH gene was used for normalization of gene expression, at ${ }^{*} \mathrm{P}<0.05 ;{ }^{*} \mathrm{P}<0.01$; ***P $<0.001$ compared with MCF-7 cells based on Student's t test.

\section{Results}


198

199

200

201

202

203

204

205

206

207

208

209

210

211

212

213

214

215

216

217

218

219

220

221

222

223

224

225

226

227

228

229

230

231

232

233

234

235

236

237

238

\section{Identification of DEGs}

The research process of bioinformatics analysis is displayed in Figure 1. We identified 865 significant DEGs, consisting of 399 upregulated and 466 downregulated DEGs, between MCF-7 cell lines and tamoxifen-resistant MCF-7/TR cell lines based on the public microarray dataset GSE26459. Among them, the most significantly upregulated gene was $W N T 2 B$, and the most significantly downregulated gene was $A L C A M$. The top ten downregulated and upregulated DEGs are presented in Table 1.

\section{DEG Gene Ontology and pathway enrichment analysis}

We clustered the DEGs through GO and KEGG pathway analyses in DAVID to explore potential biological roles and functional enrichment. The enriched GO terms, including BP, CC and MF, are presented in Fig. 2A, 2B and 2C, respectively. The most significantly enriched BP GO terms were 'telomere organization' (GO, 1903047), 'nucleosome assembly' (GO, 0044839) and 'chromatin silencing at rDNA' (GO, 0000086) (Fig. 2A). In the CC category (Fig. 2B), cell membrane components were the major enriched categories, which included extracellular exosomes (173 genes), protein complexes (41 genes) and extracellular matrices ( 34 genes). Other enriched GO terms consisted of membrane (124 genes) and cell-cell adherens junctions (33 genes, Fig. 2B). In the MF category (Fig. 2C), the most important GO categories were bindingrelated items for protein heterodimerization activity (60 genes) and histone binding ( 29 genes, Fig. 2C).

Moreover, KEGG pathway analysis showed enrichment of 5 key pathways, including antigen processing and presentation signaling ( 8 genes), allograft rejection ( 6 genes) and cell adhesion molecules (CAMs) (11 genes, Table 2).

Hub genes in STRING analysis of tamoxifen resistance were used to identify the PPI of the DEGs. PPI information for the 865 DEGs was evaluated in the STRING website, and we then used Cytoscape software to establish the PPI network for the top 15 DEGs, which contained 108 nodes and 226 edges.

The hub genes were evaluated by CytoHubba. In the PPI networks, 10 node proteins-ACLY, HSPD1, PFAS, GART, TXN, HSPH1, HSPE1, IRAS, TRAP1 and ATIC - were associated strongly with other node proteins (more than 5), suggesting a high degree of connectivity (Fig. 3). Consistently, RT-qPCR analysis indicated that the expression level of these 10 genes was increased in MCF-7/TR (tamoxifen-resistant cell line) cells comparing with MCF-7 (tamoxifen-sensitive cell line) cells (Fig. 4). Collectively, it's indicated that these hub genes and proteins may play a critical role in tamoxifen resistance.

\section{Tamoxifen resistance-associated hub genes predict poor prognosis}

For survival analysis, we evaluated associations between expression of the 10 hub genes and patient survival with the Kaplan-Meier Plotter database. Four hub genes (TXN, HSPDI, HSPHI and $A T I C$ ) were found to be related to poor overall survival in breast cancer patients who received tamoxifen (Fig. 5).

\section{Knockdown of target genes by siRNAs}

MTS assay verified that MCF-7/TR cell line (IC50 17.21 $\mu \mathrm{M}$ ) was less sensitive to tamoxifen

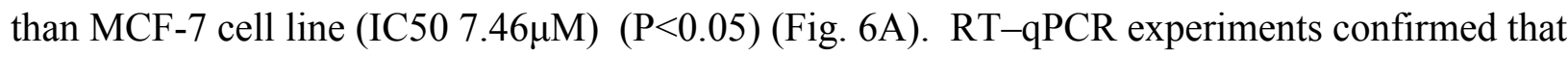


239 small interfering RNA (siRNA) resulted in over 60\% reduction in expression of 10 hub genes

240 (Fig. 6B). MTS assay showed that knockdown of HSPH1 may lead to reduced growth of MCF-

$2417 /$ TR cell $(\mathrm{P}=0.07)$, whereas knockdown of other genes did not significantly affect cell growth

242 (Fig. 6C). It's indicated that upregulation of HSPH1 is likely to play an important role in

243 tamoxifen resistance.

244

\section{Discussion}

246 Tamoxifen resistance is an unsolved problem in breast cancer treatment, hence it is particularly

247 important to identify critical genes and signaling pathways related to tamoxifen resistance to

248 determine its complex mechanism. In the present study, we identified 865 DEGs, including 399

249 upregulated and 466 downregulated DEGs, between the MCF-7 and MCF-7/TR cell lines. These

250 DEGs possibly mediated tamoxifen resistance. Previous findings suggested that several DEGs

251 might be responsible for resistance in diverse cancers. For example, $W N T 2 B$, among the top ten

252 upregulated DEGs, is important in chemotherapy resistance and tumorigenesis in head and neck

253 squamous cell carcinoma and may be a treatment target for oral cancers ( $\mathrm{Li}$, Yang \& Qian,

254 2015). The present study identified SLC6A14 as a new treatment target for ER-positive breast

255 cancer. It's indicated that SLC6A14 may play a role in tamoxifen resistance (Karunakaran et al.,

256 2011). COX6C, listed in the top ten upregulated DEGs, affects mitochondrial genes and function

257 and induces $A B C G 2$-overexpressing cells to pump mitoxantrone from the cell matrix (Chang et

258 al. 2017). $A L C A M$, among the top ten downregulated DEGs, is a new serum marker correlating

259 with cell growth, adhesion and chemotherapy resistance in pancreatic cancer (Hong et al., 2010).

260 In addition, the miR-20a-5p/SDC2 axis is a possible biomarker for diagnosis and treatment

261 targets (Zhao et al., 2017). In conclusion, these DEGs are likely to play vital roles in tamoxifen

262 resistance via various mechanisms.

263 We explored the biological functions of the DEGs with GO analysis. In the BP category, the

264 DEGs were mostly enriched in telomere organization; other DEGs were enriched in

265 nonmembrane-related items, including nucleosome assembly and chromatin silencing at rDNA.

266 Regarding the $\mathrm{CC}$ category, stimulus-related items, including extracellular exosomes, were most

267 significant. Other enriched categories included protein complexes and extracellular matrix. These

268 data showed that the molecular mechanisms of tamoxifen resistance might be related to both

269 membrane and nonmembrane structures. Binding-related items for protein heterodimerization

270 activity were most significant in the MF category, and other enriched MF terms included histone

271 binding and nucleosomal DNA binding.

272 Analysis of pathways may uncover more exact biological functions of DEGs than those obtained

273 through Gene Ontology analysis. We identified three enriched pathways: antigen processing and

274 presentation signaling pathways, pathways in allograft rejection and cell adhesion molecules.

275 Cancer pathways may participate in cancer evolution, including drug resistance.

276 Our PPI network included ACLY, HSPD1, PFAS, GART, TXN, HSPH1, HSPE1, IRAS, TRAP1,

277 and $A T I C$, which were confirmed by RT-qPCR, and several of them are associated with drug

278 resistance. Four hub genes ( $T X N, H S P D 1, H S P H 1$ and $A T I C$ ) were related to OS in patients who 
279

280

281

282

283

284

285

286

287

288

289

290

291

292

293

294

295

296

297

298

299

300

301

302

303

304

305

306

307

308

309

310

311

312

313

314

315

316

317

318

accepted tamoxifen treatment according to Kaplan-Meier Plotter. It's indicated that the overexpression of TXN proteins was one of the atypical multidrug resistance mechanisms associated with overexpression of P-glycoprotein (Mieszala et al., 2018). TXN modulate the expression of estrogen responsive genes in breast cancer cells, and reducing oxidized TXN restore tamoxifen sensitivity to previously resistant breast cancer cells (Rosalind \& Deodutta, 2013). A previous study demonstrated that the expression level of the HSPD1 mRNA was closely related to the emergence of cisplatin resistance in head and neck cancer cell lines (Nakata et al., 1994). In addition, high transcript level of HSPD1 was associated with the resistance to hormonal therapy, and a short 4-gene signature including HSPD1 might effectively predict tamoxifen-resistance (Sotgia et al., 2017). The function of HSPH1 in DNA repair may partially explain the higher resistance to genotoxic drugs of colorectal cancers expressing higher levels of HSPH1 (Causse et al., 2018). ATIC was associated with resistance to methotrexate (You et al., 2013). We further knocked down 10 hub genes by siRNAs and found silence of HSPH1 led to reduced growth of MCF-7/TR cell with a tendency toward statistical significance $(\mathrm{P}=0.07)$. Upregulation of HSPH1 may be important in the mechanism of tamoxifen resistance.

Several limitations of the present study merit discussion, including the fact that only the GEO26459 dataset of microarray data was used and that the sample size was relatively small. The aforementioned results, including the DEGs and their functions, should be confirmed by in vivo or in vitro experiments, which will be carried out in future studies. Nonetheless, the preliminary findings of our study provide comprehensive molecular insight and potential directions for further elucidating the underlying mechanisms of tamoxifen resistance.

\section{Conclusions}

Our study revealed a number of critical hub genes which might play an important role in the mechanism of tamoxifen resistance. These hub genes, including TXN, HSPD1, HSPH1, and $A T I C$, serve as potential therapeutic targets in breast cancer resistant to tamoxifen and suggest directions for uncovering the mechanisms of tamoxifen resistance. The study results have provided a theoretical basis for future translational research, which may eventually solve the clinical problem of tamoxifen resistance.

\section{Acknowledgements}

We thank all authors for their cooperation.

\section{References}

Badia E, Duchesne MJ, Semlali A, Fuentes M, Giamarchi C, Richard-Foy H, Nicolas JC, Pons M. 2000. Long-term hydroxytamoxifen treatment of an MCF-7-derived breast cancer cell line irreversibly inhibits the expression of estrogenic genes through chromatin remodeling. Cancer research, 60:4130-4138. DOI:10.1097/00002820-200008000-00010.

Baum M, Budzar AU, Cuzick J, Forbes J, Houghton JH, Klijn JG, Sahmoud T. 2002.

Anastrozole alone or in combination with tamoxifen versus tamoxifen alone for adjuvant 
319 treatment of postmenopausal women with early breast cancer: first results of the ATAC

320 randomised trial. Lancet, 359: 2131-2139. DOI: 10.1016/s0140-6736(02)09088-8.

321 Causse SZ, Marcion G, Chanteloup G, Uyanik B, Boudesco Ch, Grigorash BB, Douhard R, Dias

322 AMM, Dumetier B, Dondaine L, Gozzi GJ, Moussay E, Paggetti J, Mirjolet C, de

323 Thonel A, Dubrez L, Demidov ON, Gobbo J, Garrido C. 2018. HSP110 translocates to the

324 nucleus upon genotoxic chemotherapy and promotes DNA repair in colorectal cancer cells.

325 Oncogene, 38: 2767-2777. DOI: 10.1038/s41388-018-0616-2

326 Chang FW, Fan HC, Liu JM, Fan TP, Jing J, Yang CL, Hsu RJ. 2017. Estrogen Enhances the

327 Expression of the Multidrug Transporter Gene ABCG2-Increasing Drug Resistance of Breast

328 Cancer Cells through Estrogen Receptors. International journal of molecular sciences, 18: E163.

329 DOI: 10.3390/ijms18010163.

330 Chin $\mathrm{CH}$, Chen SH, Wu HH, Ho CW, Ko MT, Lin CY. 2014. CytoHubba: identifying hub

331 objects and sub-networks from complex interactome. BMC systems biology, 8:S11. DOI:

332 10.1186/1752-0509-8-S4-S11.

333 Early Breast Cancer Trialists' Collaborative Group. 1998. Tamoxifen for early breast cancer: an

334 overview of the randomised trials. Early Breast Cancer Trialists' Collaborative Group. Lancet,

335 351:1451-1467. DOI: https://doi.org/10.1016/S0140-6736(97)11423-4.

336 Gutierrez MC, Detre S, Johnston S, Mohsin SK, Shou J, Allred DC, Schiff R, Osborne CK, 337 Dowsett M. 2005. Molecular changes in tamoxifen-resistant breast cancer: relationship between 338 estrogen receptor, HER-2, and p38 mitogen-activated protein kinase. Journal of clinical 339 oncology: official journal of the American Society of Clinical Oncology, 23:2469-2476. DOI: 340 10.1200/JCO.2005.01.172.

341 Hong X, Michalski CW, Kong B, Zhang W, Raggi MC, Sauliunaite D, De Oliveira T, Friess H,

342

343

344

345

346 Kleeff J. 2010. ALCAM is associated with chemoresistance and tumor cell adhesion in pancreatic cancer. Journal of surgical oncology, 101:564-569. DOI: 10.1002/jso.21538.

Huang da W, Sherman BT, Lempicki RA. 2009. Systematic and integrative analysis of large gene lists using DAVID bioinformatics resources. Nature protocols, 4: 44-57. DOI: 10.1038/nprot.2008.211.

347 Karunakaran S, Ramachandran S, Coothankandaswamy V, Elangovan S, Babu E, Periyasamy348 Thandavan S, Gurav A, Gnanaprakasam JP, Singh N, Schoenlein PV, Prasad PD, Thangaraju M, 349 Ganapathy V. 2011. SLC6A14 (ATB0,+) protein, a highly concentrative and broad specific amino acid transporter, is a novel and effective drug target for treatment of estrogen receptorpositive breast cancer. The Journal of biological chemistry, 286:31830-31838. DOI:

352 10.1074/jbc.M111.229518.

Katzenellenbogen BS, Frasor J. 2004. Therapeutic targeting in the estrogen receptor hormonal pathway. Seminars in oncology, 31:28-38. DOI: 10.1053/j.seminoncol.2004.01.004.

Knowlden JM, Hutcheson IR, Jones HE, Madden T, Gee JM, Harper ME, Barrow D, Wakeling AE, Nicholson RI. 2003. Elevated levels of epidermal growth factor receptor/c-erbB2 heterodimers mediate an autocrine growth regulatory pathway in tamoxifen-resistant MCF-7 cells. Endocrinology, 144:1032-1044. DOI: 10.1210/en.2002-220620.

360

361 Li SJ, Yang X, Qian HY. 2015. Antitumor effects of WNT2B silencing in GLUT1 overexpressing cisplatin resistant head and neck squamous cell carcinoma. American journal of cancer research, 5:300-308. DOI: http://dx.doi.org.

362 Mieszala K, Rudewicz M, Gomulkiewicz A, Ratajczak-Wielgomas K, Grzegrzolka J, Dziegiel P, 363 Borska S. 2018. Expression of genes and proteins of multidrug resistance in gastric cancer cells 364 treated with resveratrol. Oncology Letters, 15: 5825-5832. DOI: 10.3892/ol.2018.8022. 
365 Nakata B, Barton RM, Robbins KT, Howell SB, Los G. 1994. Association between hsp60

366 messenger-RNA levels and Cisplatin resistance in human head and neck-cancer cell-lines.

367 International Journal of Oncology, 5:1425-1432. DOI: 10.3892/ijo.5.6.1425.

368 Nass N, Kalinski T. 2015. Tamoxifen resistance: from cell culture experiments towards novel

369 biomarkers. Pathology, research and practice, 211:189-197. DOI: 10.1016/j.prp.2015.01.004.

370 Normanno N, Di Maio M, De Maio E, De Luca A, de Matteis A, Giordano A, Perrone F. 2005.

371 Mechanisms of endocrine resistance and novel therapeutic strategies in breast cancer. Endocrine-

372 related cancer, 12: 721-747. DOI: 10.1677/erc.1.00857.

373 Ring A, Dowsett M. 2004. Mechanisms of tamoxifen resistance. Endocrine-related 374 cancer, 11:643-658. DOI: 10.1677/erc.1.00776.

375 Ritchie ME, Phipson B, Wu D, Hu Y, Law CW, Shi W, Smyth GK. 2015. limma powers 376 differential expression analyses for RNA-sequencing and microarray studies. Nucleic acids 377 research, 43: e47. DOI: 10.1093/nar/gkv007.

378 Rondon-Lagos M, Villegas VE, Rangel N, Sánchez MC, Zaphiropoulos PG. 2016. Tamoxifen 379 Resistance: Emerging Molecular Targets. International journal of molecular sciences, 17:E1357. 380 DOI: $10.3390 / \mathrm{ijms} 17081357$.

381 Rosalind BP, Deodutta R. 2013. Thioredoxin-mediated redox regulation of resistance to 382 endocrine therapy in breast cancer. Biochimica et biophysica acta, 1836:60-79. DOI: 383 10.1016/j.bbcan.2013.02.005.

384 Schmid R, Blaxter ML. 2008. annot8r: GO, EC and KEGG annotation of EST datasets. BMC 385 bioinformatics, 9:180. DOI: 10.1186/1471-2105-9-180.

386 Shang Y, Brown M. 2002. Molecular determinants for the tissue specificity of SERMs. Science, 387 295:2465-2468. DOI: 10.1126/science.1068537.

388 Shannon P, Markiel A, Ozier O, Baliga NS, Wang JT, Ramage D, Amin N, Schwikowski B, 389 Ideker T. 2003. Cytoscape: a software environment for integrated models of biomolecular 390 interaction networks. Genome research, 13: 2498-2504. DOI: 10.1101/gr.1239303.

391 Sotgia F, Fiorillo M, Lisanti MP. 2017. Mitochondrial markers predict recurrence, metastasis and 392 tamoxifen-resistance in breast cancer patients: Early detection of treatment failure with 393 companion diagnostics. Oncotarget, 8:68730-68745. DOI: 10.18632/oncotarget.19612.

394 Szklarczyk D, Franceschini A, Wyder S, Forslund K, Heller D, Huerta-Cepas J, Simonovic M, 395 Roth A, Santos A, Tsafou KP, Kuhn M, Bork P, Jensen LJ, von Mering C. 2015. STRING v10: 396 protein-protein interaction networks, integrated over the tree of life. Nucleic acids research, 43: 397 D447-452. DOI: 10.1093/nar/gku1003.

398 Torre LA, Bray F, Siegel RL, Ferlay J, Lortet-Tieulent J, Jemal A. 2015. Global cancer statistics, 399 2012. CA: a cancer journal for clinicians, 65:87-108. DOI: 10.3322/caac.21262.

400 Turner NC, Neven P, Loibl S, Andre F. 2017. Advances in the treatment of advanced oestrogen401 receptor-positive breast cancer. Lancet, 389:2403-2414. DOI: 10.1016/S0140-6736(16)32419-9.

402 Viedma-Rodriguez R, Baiza-Gutman L, Salamanca-Gomez F, Diaz-Zaragoza M, Martínez-

403 Hernández G, Ruiz Esparza-Garrido R, Velázquez-Flores MA, Arenas-Aranda D. 2014.

404 Mechanisms associated with resistance to tamoxifen in estrogen receptor-positive breast cancer

405 (review). Oncology reports, 32:3-15. DOI: 10.3892/or.2014.3190.

406 You X, Williams A, Dervieux T, He WJ, Cronstein BN. 2013. Fibroblasts from methotrexate407 sensitive mice accumulate methotrexate polyglutamates but those from methotrexate-resistant 408 mice do not. Clinical \& Experimental Rheumatology, 31:433-435. DOI:

409 10.1016/j.jbspin.2012.09.027.

410 Zhang L, Huang Y, Zhuo W, Zhu Y, Zhu B, Chen Z. 2017. Identification and characterization of 
411 biomarkers and their functions for Lapatinib-resistant breast cancer. Medical oncology, 34:89. 412 DOI: $10.1007 / \mathrm{s} 12032-017-0953-\mathrm{y}$.

413 Zhao F, Pu Y, Cui M, Wang H, Cai S. 2017. MiR-20a-5p represses the multi-drug resistance of 414 osteosarcoma by targeting the SDC2 gene. Cancer cell international, 17:100. DOI: 415 10.1186/s12935-017-0470-2.

416 Zhu Y, Liu Y, Zhang C, Chu J, Wu Y, Li Y, Liu J, Li Q, Li S, Shi Q, Jin L, Zhao J, Yin 417 D, Efroni S, Su F, Yao H, Song E, Liu Q. 2018. Tamoxifen-resistant breast cancer cells are 418 resistant to DNA-damaging chemotherapy because of upregulated BARD1 and BRCA1. Nature 419 communications, 9:1595. DOI: 10.1038/s41467-018-03951-0. 


\section{Table $\mathbf{1}$ (on next page)}

Table 1

Table 1 The most significant up-regulated and down-regulated DEGs (top ten, Tamoxifenresistant MCF-7/TR versus Tamoxifen-sensitive MCF-7) 
1 Table 1 The most significant up-regulated and down-regulated

2 DEGs (top ten, Tamoxifen-resistant MCF-7/TR versus Tamoxifen-sensitive MCF-7)

\begin{tabular}{|c|c|c|c|c|}
\hline Gene & $\log \mathrm{FC}$ & P.Value & Gene full name & Gene ID number \\
\hline \multicolumn{5}{|c|}{ Up-regulated } \\
\hline WNT2B & 5.315 & $7.3 \times 10^{-12}$ & Wnt Family Member 2B & $\underline{7482}$ \\
\hline EVL & 5.292 & $4.1 \times 10^{-11}$ & Enah/Vasp-Like & $\underline{51466}$ \\
\hline TBC1D9 & 2.331 & $1.1 \times 10^{-10}$ & $\begin{array}{ll}\text { TBC1 Domain } & \text { Family } \\
\text { Member } 9 & \end{array}$ & $\underline{23158}$ \\
\hline LMCD1 & 2.862 & $1.1 \times 10^{-10}$ & $\begin{array}{l}\text { LIM And Cysteine Rich } \\
\text { Domains } 1\end{array}$ & 29995 \\
\hline CSTA & 3.675 & $1.5 \times 10^{-10}$ & Cystatin A & $\underline{1475}$ \\
\hline SLC6A14 & 3.391 & $5.9 \times 10^{-10}$ & $\begin{array}{lll}\text { Solute Carrier } & \text { Family } & 6 \\
\text { Member } 14 & & \end{array}$ & $\underline{11254}$ \\
\hline PODXL & 2.196 & $7.1 \times 10^{-10}$ & Podocalyxin Like & $\underline{5420}$ \\
\hline COX6C & 2.163 & $1.2 \times 10^{-09}$ & $\begin{array}{lll}\text { Cytochrome } & \text { C } & \text { Oxidase } \\
\text { Subunit 6C } & & \\
\end{array}$ & $\underline{1345}$ \\
\hline TNS3 & 2.431 & $1.3 \times 10^{-09}$ & Tensin 3 & $\underline{64759}$ \\
\hline ACSL1 & 2.406 & $1.3 \times 10^{-09}$ & $\begin{array}{l}\text { Acyl-CoA Synthetase } \\
\text { Long Chain Family } \\
\text { Member } 1\end{array}$ & $\underline{2180}$ \\
\hline \multicolumn{5}{|c|}{ Down-regulated } \\
\hline ALCAM & -5.393 & $3.7 \times 10^{-12}$ & $\begin{array}{l}\text { Activated Leukocyte Cell } \\
\text { Adhesion Molecule }\end{array}$ & $\underline{214}$ \\
\hline
\end{tabular}




\begin{tabular}{|c|c|c|c|c|}
\hline LXN & -4.032 & $7.3 \times 10^{-12}$ & Latexin & $\underline{56925}$ \\
\hline TARP & -5.160 & $2.1 \times 10^{-11}$ & $\begin{array}{l}\text { TCR Gamma Alternate } \\
\text { Reading Frame Protein }\end{array}$ & $\underline{445347}$ \\
\hline TRGC2 & -5.409 & $3.7 \times 10^{-11}$ & $\begin{array}{l}\text { T Cell Receptor Gamma } \\
\text { Constant } 2\end{array}$ & 6967 \\
\hline РPP3CA & -2.883 & $1.8 \times 10^{-10}$ & $\begin{array}{l}\text { Protein Phosphatase } 3 \\
\text { Catalytic Subunit Alpha }\end{array}$ & 5530 \\
\hline CLEC7A & -2.609 & $2.8 \times 10^{-10}$ & $\begin{array}{l}\text { C-Type Lectin Domain } \\
\text { Containing 7A }\end{array}$ & 64581 \\
\hline SOWAHA & -2.190 & $5.8 \times 10^{-10}$ & $\begin{array}{l}\text { Sosondowah Ankyrin } \\
\text { Repeat Domain Family } \\
\text { Member A }\end{array}$ & $\underline{134548}$ \\
\hline CD36 & -3.636 & $6.3 \times 10^{-10}$ & CD36 Molecule & $\underline{948}$ \\
\hline CLEC3A & -2.534 & $6.8 \times 10^{-10}$ & $\begin{array}{l}\text { C-Type Lectin Domain } \\
\text { Family } 3 \text { Member A }\end{array}$ & 10143 \\
\hline SDC2 & -2.583 & $8.2 \times 10^{-10}$ & Syndecan 2 & 6383 \\
\hline
\end{tabular}

3

4

5

6

7

8

9

10

11

12

13

14

15

16 
Table 2 (on next page)

The enriched KEGG pathway of DEGs 
1 Table 2 The enriched KEGG pathway of DEGs

2

3

\begin{tabular}{|l|l|l|l|}
\hline Term & Description & Count & PValue \\
\hline hsa04612 & $\begin{array}{l}\text { Antigen processing } \\
\text { and presentation }\end{array}$ & 8 & $9.71 \mathrm{E}-04$ \\
\hline hsa05330 & Allograft rejection & 6 & $9.34 \mathrm{E}-04$ \\
\hline hsa04514 & $\begin{array}{l}\text { Cell adhesion } \\
\text { molecules (CAMs) }\end{array}$ & 11 & $7.19 \mathrm{E}-04$ \\
\hline hsa05332 & $\begin{array}{l}\text { Graft-versus-host } \\
\text { disease }\end{array}$ & 6 & $5.44 \mathrm{E}-04$ \\
\hline hsa04940 & $\begin{array}{l}\text { Type I diabetes } \\
\text { mellitus }\end{array}$ & 7 & $2.09 \mathrm{E}-04$ \\
\hline
\end{tabular}




\section{Figure 1}

Figure 1

The flowchart of the bioinformatics analysisDEGs, differentially expressed genes; GO, Gene Ontology; KEGG, Kyoto Encyclopedia of Genes and Genomes; PPI, protein-protein interaction; K-M Plotter, Kaplan-Meier Plotter; RT-qPCR, quantitative real-time polymerase chain reaction; MTS, 3-(4,5-dimethylthiazol-2-yl)-5-(3-carboxymethoxyphenyl)-2-(4-sulfophenyl)-2Htetrazolium, inner salt assay. 


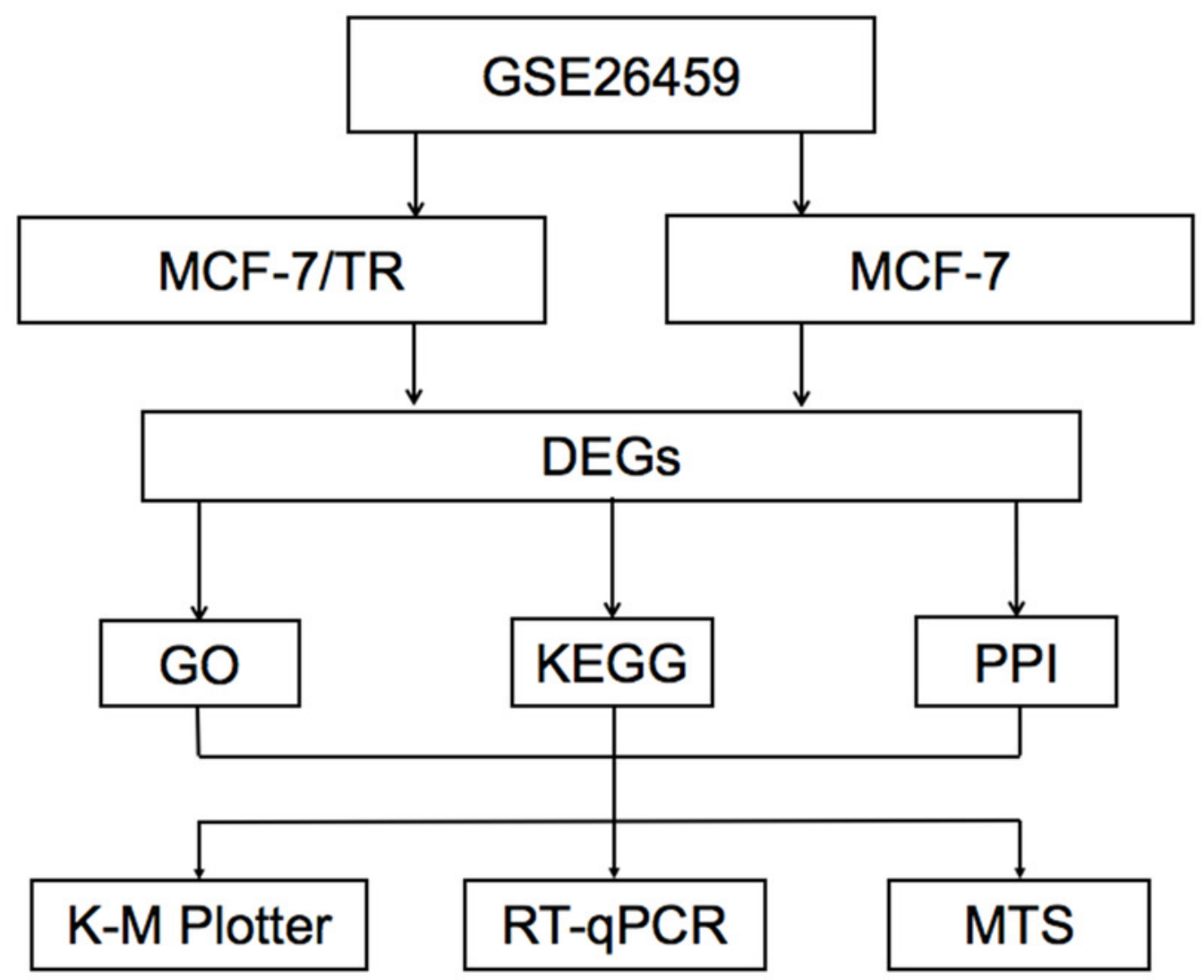


Figure 2

Figure 2

GO enrichment of DEGsA. in biological process ontology; B. in cellular component ontology; C. in molecular function ontology. 

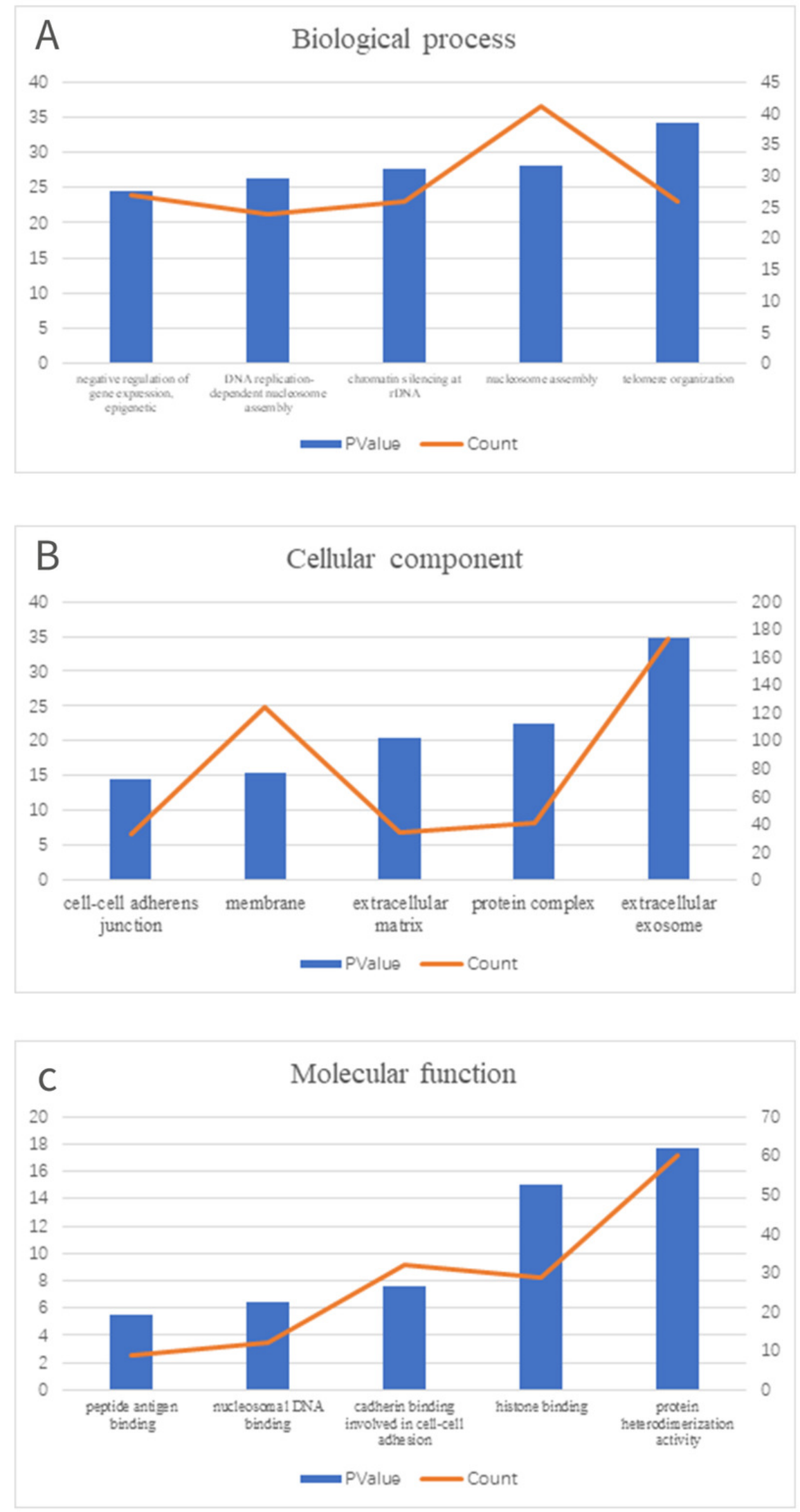
Figure 3

Figure 3

The PPI network diagram of the node proteins showed lots of associations with other node proteins, indicating that they have high degrees of connectivity. The darker the color was, the more proteins associated with the node protein.

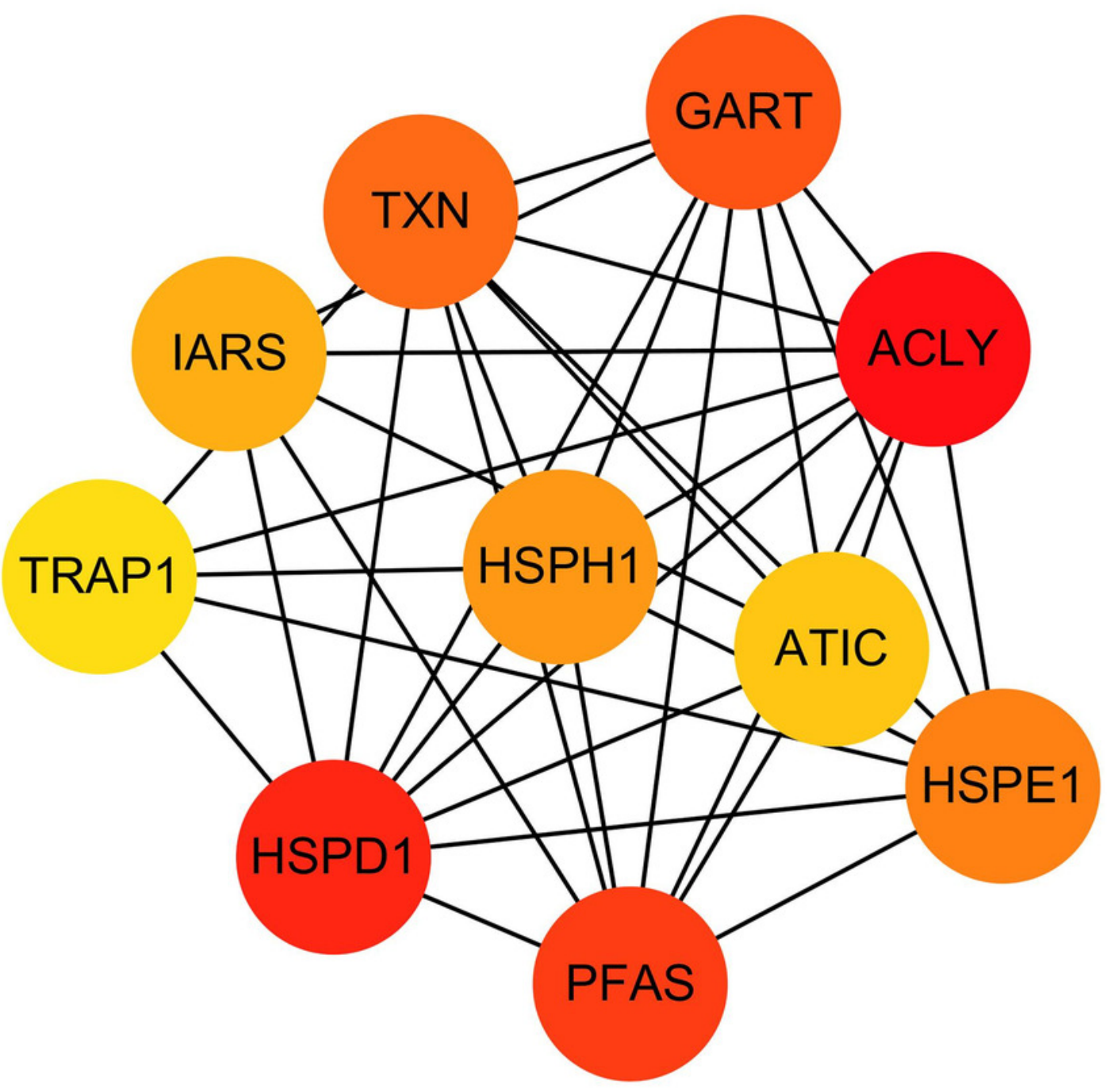


Figure 4

Figure 4

RT-qPCR validation of the DEGs between MCF-7 and MCF-7/TR. Relativeexpression of ACLY, HSPD1, PFAS, GART, TXN, HSPH1, HSPE1, IRAS, TRAP1, and ATIC. *P < 0.05; **P < 0.01; ***P $<0.001$

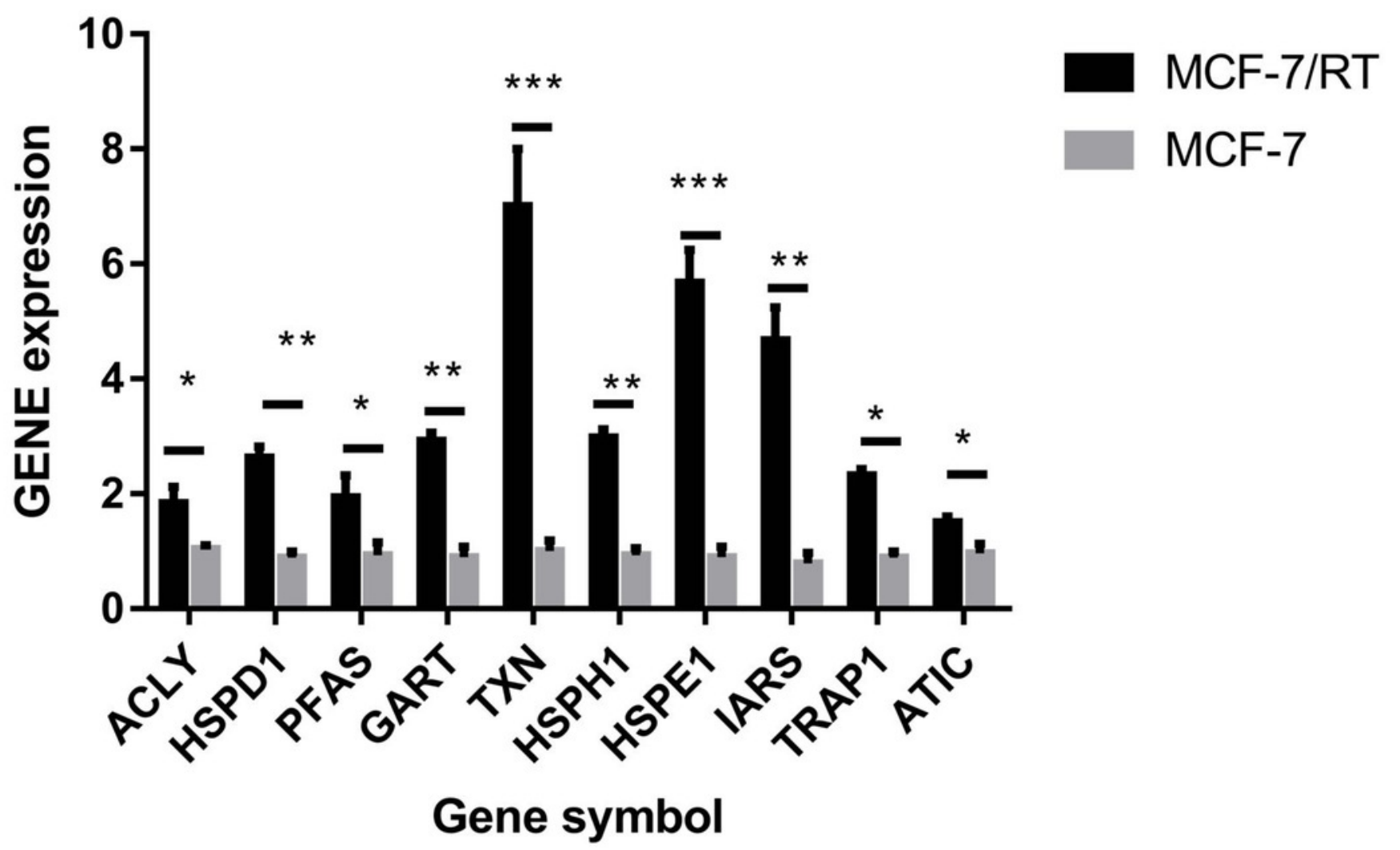


Figure 5

Figure 5

Kaplan-Meier Plotter analysis of four genes involved in breast cancer

A: TXN; B: HSPD1; C: HSPH1; D: ATIC.

HR: Hazard ratio; $\mathrm{Cl}$ : confidence interval.
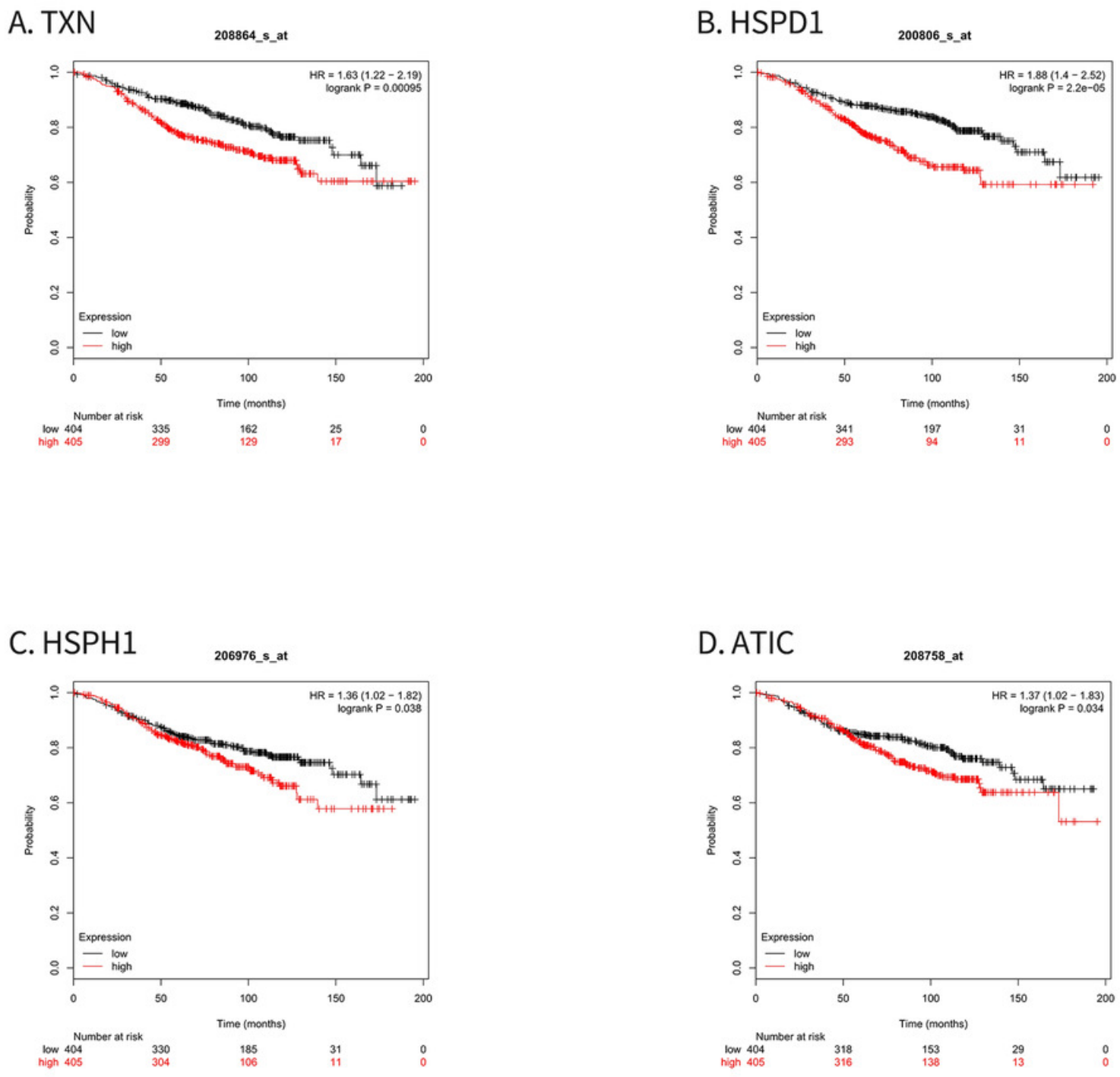


\section{Figure 6}

Figure 6

(A) MTS assay verified that MCF-7/TR cell line (IC50 17.21 $\mu \mathrm{M})$ was less sensitive to tamoxifen

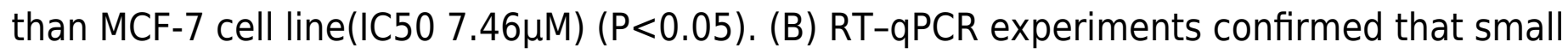
interfering RNA (siRNA) resulted in over $60 \%$ reduction in expression of 10 hub genes. (C) MTS assay showed that knockdown of HSPH1 may lead to reduced growth of MCF-7/TR cell with a trend close to significance $(P=0.07)$, whereas knockdown of other genes did not significantly affect cell growth. 


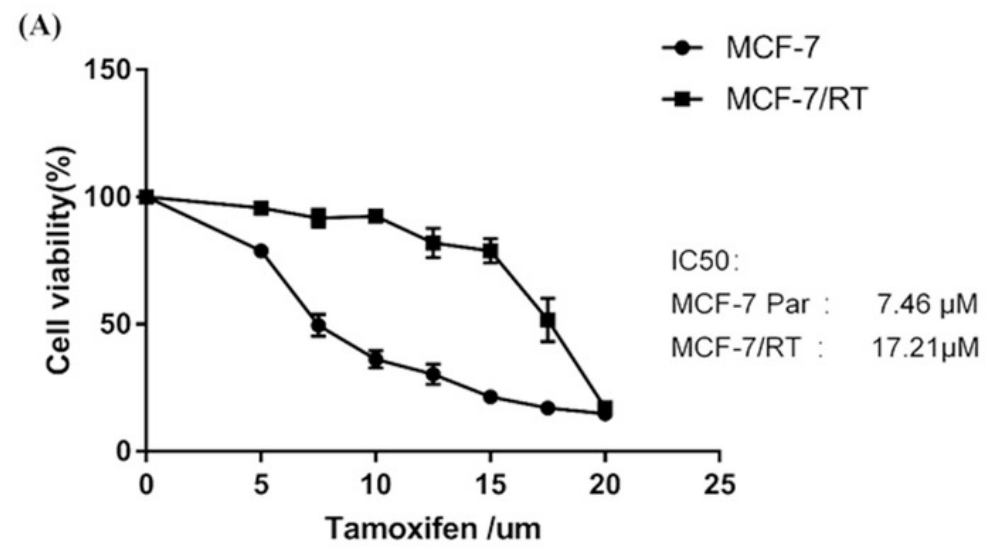

(B)

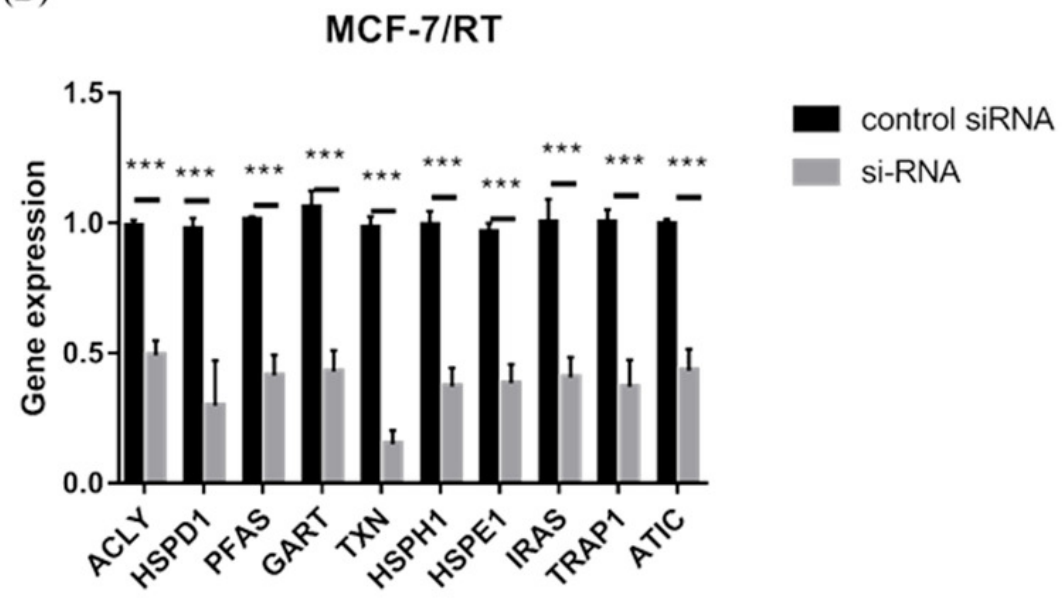

(C)

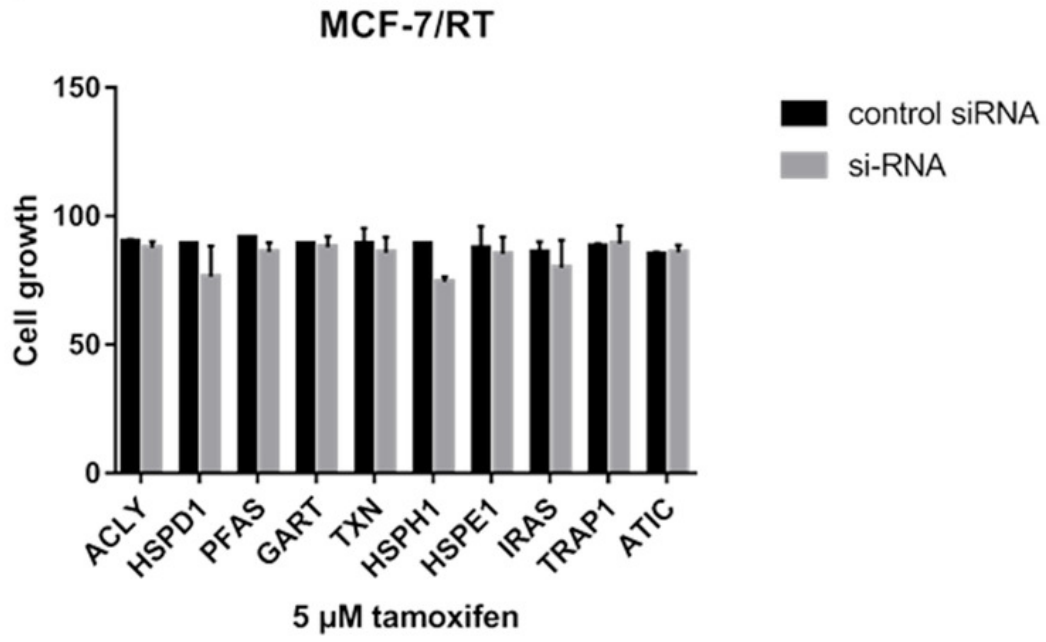

